\title{
Evaluación fisicoquímica y microbiológica del aperitivo vínico de lulo (Solanum quitoense $L$.)
}

\author{
Clemente Granados $^{(1) \star}$, Miladys E. Torrenegra ${ }^{(1)}$, Diofanor Acevedo ${ }^{(1)}$ y Pedro Romero ${ }^{(2)}$
}

(1) Universidad de Cartagena, Facultad de Ingeniería, Programa de ingeniería de Alimentos, Avenida el Consulado, Calle 30 No. 48-152. Cartagena, Bolívar-Colombia (e-mail: clementecondeg@gmail.com)

(2) Universidad de Córdoba, Facultad de Ciencias Agrícolas, Departamento de Ingeniería de Alimentos, Carrera 6 No 76-103, Km. 3, vía Cereté, Córdoba-Colombia (e-mail: rtorres@correo.unicordoba.edu.co)

* autor a quien debe ser dirigida la correspondencia

Recibido Abr. 17, 2013; Aceptado May. 29, 2013; Versión final recibida Jul. 15, 2013

\section{Resumen}

Se ha estudiado una formulación que incluye pulpa de lulo con el fin de aprovechar este fruto para la obtención de aperitivos vínicos. Se utilizó la siguiente formulación: Pulpa de lulo (1\%), agua (80,5\%), azúcar $(18 \%)$, ácido cítrico $(0,03 \%)$, fosfato diamónico $(0,03 \%)$ y levadura $(0,42 \%)$. Los análisis microbiológicos realizados estuvieron dentro de los rangos permitidos. La fermentación se detuvo a los diez días, y luego se determinaron análisis fisicoquímicos. Posteriormente se clarificó durante cinco días. La acidez titulable fue de $0,06 \%$, la acidez volátil de $0,11 \%$ y el $\mathrm{pH}$ de 3,24 . Se obtuvo una bebida con un contenido alcohólico de 11,3 grados $\left({ }^{\circ} \mathrm{GL}\right)$, lo cual es suficiente para ser considerada como un aperitivo vínico de calidad comparable con la de los productos existentes en el mercado. El rendimiento para la pulpa fue de $66,7 \%$.

Palabras clave: aperitivo vínico, pulpa de lulo, fermentación, grados de alcohol

\section{Physicochemical and microbiological evaluation vinous of pineapple appetizer (Solanum quitoense L.)}

\begin{abstract}
A formulation including lulo pulp aiming to use this fruit to produce vinous snacks was studied. The following formulation was employed: Lulo pulp $(1 \%)$, water $(80.5 \%)$, sugar $(18 \%)$, citric acid $(0.03 \%)$, diammonium phosphate $(0.03 \%)$ and yeast $(0.42 \%)$. Microbiological analyzes were performed and were found within allowable ranges. The fermentation was stopped after ten days, and then physicochemical analyses were done. After that the product was clarified during five days. Titratable acidity was $0.06 \%$, volatile acidity was $0.11 \%$ and $\mathrm{pH}$ 3.24. A beverage with an alcohol content of 11,3 percent alcohol ( ${ }^{\circ} \mathrm{GL}$ ) was obtained. This alcoholic level is enough to be considered as a vinous appetizer of quality comparable to existing products. The yield for the pulp was $66.7 \%$.
\end{abstract}

Keywords: vinous appetizer, lulo pulp, fermentation, alcoholic degrees. 


\section{INTRODUCCIÓN}

El fruto del Solanum quitoense L., se conoce popularmente en Colombia como lulo. Esta planta tiene un promedio de $2 \mathrm{~m}$ de altura y su cultivo se da principalmente en climas templados entre los 17 y $20^{\circ} \mathrm{C}$, y a una altura de 1,2 a $2 \mathrm{~m}$ sobre el nivel del mar. Este fruto goza de un gran mercado internacional, en el cual Colombia, junto con el Ecuador, son los mayores productores, estimándose que juntos pueden alcanzar una producción anual de 100.000 toneladas. Por su parte, Estados Unidos es el mayor comprador mundial (Fundación CODESARROLLO, 2006).

El árbol de lulo es poco conocido, y es cultivado en Colombia, Costa Rica, Ecuador, Honduras, Panamá y Perú (Flórez et al., 2008; Pulido et al., 2008; Acosta et al., 2009). Este se cultiva exclusivamente en zonas tropicales donde se produce normalmente durante todo el año, con una gran variabilidad en el rendimiento en el espacio y el tiempo. Para el caso de colombiano, el cultivo es en condiciones muy heterogéneas (Jiménez et al., 2011). El fruto es de color verde, cuando está viche y varia de amarillo a rojo opaco cuando alcanza la madurez fisiológica, su epidermis es fina, suave y contiene cuatro celdas llenas de semillas envueltas en un mucílago claro, con un número promedio de 800 semillas por fruto, ésta tiene un pH acido entre 3,5 a 4,5, y puede llegar a $8 \mathrm{~cm}$ de diámetro y de 4 a $6 \mathrm{~cm}$ de largo, con un peso promedio de $180 \mathrm{a}$ 320 gramos (Arrázola, 1997).

Las características que más llaman la atención del lulo son el sabor, aroma y color atractivo de la pulpa. Junto con su contenido nutricional, en el que sobresalen las Vitaminas A y C, elementos que le confiere propiedades antioxidantes, diuréticas, regeneradoras de tejidos, por su contenido de Hierro se le atribuyen propiedades tonificantes y para el buen funcionamiento de los riñones. El jugo de lulo disuelve algunas toxinas en el organismo, y es recomendado para las personas que sufren gota, ya que disminuye la acumulación de ácido úrico en la sangre, responsable de esta enfermedad (FAO, 2006). La acidez y el contenido de sólidos solubles son los parámetros que se relacionan con el sabor del lulo, y la coloración verde de su pulpa y exquisito sabor le brinda grandes posibilidades para la agroindustria. La fruta ya madura puede procesarse con epidermis, obteniendo de esta forma mayores contenidos de minerales y fibra. Contiene agua en un porcentaje cercano al $91 \%$, y cerca del $28 \%$ de sus calorías no son significativas. Tambien presenta contenidos de grasas, proteínas, fibra, tiamina, riboflavina y niacina (Bermeo, 2005).

Un aperitivo es una bebida alcohólica que se obtiene a través de destilados, fermentados, infusiones, maceraciones, percolaciones o vino de frutas a las que se les puede adicionar o no productos alimenticios orgánicos y otros aditivos. Su graduación alcohólica será menor que la establecida para los licores por la legislación vigente. En cuanto a la clasificación de los aperitivos, se catalogan en vínicos cuando son productos elaborados con adición de más de $75 \%$ de vino, y no vínicos cuando hay adición de menos del $75 \%$. Los aperitivos vínicos deben cumplir los mismos requisitos de los vinos (Decreto 365 de 1994), y con las especificaciones establecidas en la NTC 1245 del 2004.

Teniendo en cuenta la heterogeneidad fisicoquímica y biodiversidad de los frutos producidos en Colombia, con base a lo anterior y con la finalidad de explorar un nuevo valor adicional del lulo, se realizó el presente trabajo con el objetivo de obtener una bebida con un contenido alcohólico suficiente que llegase a poseer aceptación de los consumidores, y que cumpliera con los parámetros establecidos en la normatividad vigente. A este producto obtenido se le evaluó la calidad fisicoquímica y microbiológica.

\section{METODOLOGÍA}

La transformación de la materia prima se llevó a cabo siguiendo los parámetros básicos de limpieza y desinfección contemplados en el Decreto 3075 (1997), que corresponde a la aplicación de Buenas Prácticas de Manufactura (BPM).

\section{Obtención de la pulpa de lulo}

Para la realización de ésta investigación se utilizó la pulpa extraída de lulos sanos con un índice de madurez de seis, siete unidades de ${ }^{\circ} B r i x, \mathrm{pH}$ de 3,4 y acidez de 1,3\%, expresada como ácido cítrico. Se recibió la pulpa en los tanques de fermentación para la obtención del aperitivo vínico.

\section{Análisis fisicoquímicos}

Se tomó una muestra de $100 \mathrm{ml}$ de pulpa, a la cual se le efectuaron análisis fisicoquímicos: ํㅏix, $\mathrm{pH}$, acidez titulable, y densidad por el método de Winnick (Lujan y Salcedo, 2004), metanol mediante la norma A.O.A.C. 9.107/84.972.11/90 (1990), acidez total según el método de la A.O.A.C. 11.042/84.962.12/90, acidez volátil 
y $\mathrm{pH}$ por el método de la A.O.A.C.11.043/84.964.08/90 (1990), y se compararon con los requisitos fisicoquímicos establecidos por la norma técnica colombianas NTC 708 (2005) para vinos de frutas.

\section{Mezclado de ingredientes}

Se adicionó al tanque de fermentación el agua y el azúcar necesario para alcanzar la concentración de 25 ${ }^{\circ}$ Brix, la levadura, el ácido cítrico, y luego se homogenizó. Se utilizó levadura activa seca para vinos de la marca comercial Fermivin y activadas a $30 \stackrel{\circ}{\circ}$ por 15 minutos en una solución de azúcar de $12{ }^{\circ}$ Brix.

\section{Fermentación}

Se utilizó la Saccharomyces cerevisiae y la siguiente formulación: Pulpa de lulo (1\%), agua (80,5\%), azúcar (18\%), ácido cítrico $(0,03 \%)$, fosfato diamónico $(0,03 \%)$ y levadura $(0,42 \%)$.

\section{Controles de parámetros calidad}

Se tomó una muestra de $50 \mathrm{ml}$ de mosto acondicionado para medir $\mathrm{pH}$, acidez, y grados alcohólicos ( ${ }^{\circ} \mathrm{GL}$ ).

\section{Filtración}

Esta operación se hace para separar el aperitivo vínico producido de lo que llamamos torta de levadura, o la masa celular producida que va a quedar en su mayoría decantada en el fondo del tanque. Esta filtración se hace con un filtro mecánico, con placas filtrantes K100.

\section{Clarificación}

Se agregaron enzimas pectinasas al $0,02 \%$ para hidrolizar la pectina que se encontraba en la pulpa de lulo. Se dejó actuar por 24 horas y luego se mezcló el resto de los ingredientes. Se esperó hasta obtener la clarificación deseada, por un total de cinco (5) días, y posterior a esto se realizó una nueva filtración, obteniendo un líquido transparente con olor, color, y sabor característico del aperitivo vínico de fruta. Una vez clarificado se filtró nuevamente, con un microfiltro K200 o placa de abrillantamiento distinto al de la filtración inicial pero en el mismo equipo (filtro mecánico).

\section{Envasado}

Esta operación se realizó con una empacadora manual, en botellas de vidrio con capacidades de $750 \mathrm{ml}$, de color verde con tapas plásticas, sostenidas por una capucha de alambre.

\section{Almacenamiento final}

En esta última etapa del proceso se colocaron las botellas en cajas de cartón que contenían seis unidades cada una y se mantuvieron a temperatura ambiente $\left(25^{\circ} \mathrm{C}\right)$ organizadas en estibas de madera con el fin de permitir los cambios fisicoquímicos necesarios para la transformación de los componentes del aperitivo vínico, así como aromas, sabores, olores y apariencia (Vogt et al., 1989).

\section{Análisis microbiológico}

Para los análisis microbiológicos se realizó recuento de mesófilos, mohos y levaduras con siembras por triplicados en profundidad y superficie con diluciones de $10^{-1}, 10^{-2} 10^{-3}$, las cuales fueron leídas a las 24 y 43 horas como UFC/ml.

\section{Análisis final}

Los análisis al producto final fueron acidez total, acidez volátil, acidez fija, determinación de contenido de metanol, $\mathrm{pH} \mathrm{y}^{\circ} \mathrm{GL}$.

\section{ANÁLISIS DE RESULTADOS}

Luego del proceso de despulpado del lulo se observó un porcentaje de pérdidas del $33,27 \%$, debido a que el lulo posee una alta cantidad de semillas. En la Tabla 1 se indican las cantidades de fruta utilizada, pulpa, obtenida, la cual tuvo un rendimiento del $66,73 \%$, mayor al obtenido por Camacho (1992), el cual fue de $65 \%$. 
Tabla 1: Porcentaje de obtención de pulpa de lulo

\begin{tabular}{|c|c|c|}
\hline Producto & Cantidad $(\mathrm{Kg})$ & Porcentaje (\%) \\
\hline Fruta & 11,48 & 100,00 \\
\hline Pulpa & 7,66 & 66,73 \\
\hline Epidermis y semilla & 2,50 & 21,77 \\
\hline Mermas o perdidas & 1,32 & 11,50 \\
\hline
\end{tabular}

Tabla 2: Pruebas fisicoquímicas para la pulpa de lulo

\begin{tabular}{|c|c|c|}
\hline Prueba & Método utilizado & Resultado \\
\hline $\mathrm{pH}$ & Potenciómetro & 3,21 \\
\hline Sólidos solubles totales & Refractómetro & $7^{\circ}$ Brix \\
\hline Acidez & Titulación ácido-base & $\begin{array}{c}1,3 \% \text { (ácido } \\
\text { cítrico) }\end{array}$ \\
\hline Densidad & Picnómetro & $1,085 \mathrm{~g} / \mathrm{ml}$ \\
\hline Índice de madurez & Relación sólidos soluble- acidez & 5,38 \\
\hline
\end{tabular}

Tabla 3: Análisis microbiológico

\begin{tabular}{|c|c|c|}
\hline Análisis & UFC/ml & Norma ICONTEC 404 \\
\hline Recuento de Mesófilos aerobios & 100 & $3000 / \mathrm{gr}$ \\
\hline Recuento de Hongos & 180 & $2000 / \mathrm{gr}$ \\
\hline
\end{tabular}

En los análisis fisicoquímicos a la pulpa de lulo (Tabla 2), los resultados fueron similares a los reportados por Camacho (1992), con los cual se puede indicar que el lulo es un buen sustrato para las levaduras en la obtención de aperitivo vínico. Se puede decir que en los ensayos microbiológicos realizados a la pulpa (Tabla 3), todos los valores de recuento de aerobios mesófilos y los de mohos y levaduras se encuentran dentro de los parámetros de la legislación (NTC 404, 2007), lo cual indica unas buenas prácticas de higiene durante la obtención de la pulpa (Caicedo \& Higuera, 2007).

Tabla 4: Pruebas fisicoquímicas del aperitivo vínico de lulo

\begin{tabular}{|c|c|c|}
\hline Prueba & Método utilizado & Resultado \\
\hline Grados alcohólicos $\left({ }^{\circ} \mathrm{GL}\right)$ & Ebullometría, alcoholimetría y destilado & 11,3 \\
\hline $\mathrm{pH}$ & Potenciómetro & 3,24 \\
\hline Densidad & Por Winnick & $0,97 \mathrm{~g} / \mathrm{ml}$ \\
\hline Acidez volátil & Destilación & $0,11 \%$ \\
\hline Metanol & Cromatografía de gases $(\mathrm{GC})$ & $108 \mathrm{mg} / \mathrm{L}$ \\
\hline
\end{tabular}

La fermentación se detuvo a los diez días. Luego se determinaron los análisis fisicoquímicos que se muestran en la Tabla 4. Machado de Castilhos et al., 2013, reportó en su estudio de Influencia de dos procedimientos de vinificación diferentes sobre las propiedades físico-químicas y sensoriales de vinos tintos, valores promediados de $\mathrm{pH}$ de 3,3 a 3,4; acidez volátil de 0,2 a 0,3; densidad de 0,99 y contenido alcohólico de 9,7 a 12,5. Los valores arrojados en las pruebas fisicoquímicas del aperitivo vínico de lulo son cercanos o se encuentran dentro del rango reportado por Machado de Castilhos et al., (2013) y otros autores como Bindon et al., (2013), Gómez et al., (2011) y Seung-Joo et al., (2006).

El Metanol no es un producto de la fermentación alcohólica, ya que su presencia en este tipo de bebidas se debe a la desesterificación de las pectinas estearasas presentes en las frutas. El límite permisible de éste alcohol según las normas oficiales Colombianas es de $3000 \mathrm{mg} / \mathrm{L}$ cuando se trata de bebidas fermentadas, y en el aperitivo vínico de lulo la concentración de metanol fue $108 \mathrm{mg} / \mathrm{L}$, por lo cual cumple con la norma y no representa peligros para la salud de los consumidores (Álvarez et al., 2009; Mesas y Alegre 1999). Sin embargo, estos niveles sobrepasan algunos de los datos reportados por Cabaroglu (2005), quien en su investigación para determinar los contenidos de metanol de vinos varietales de Turquía, encontró que los niveles de metanol variaron desde 30,5 hasta $121,4 \mathrm{mg} / \mathrm{L}$ en los vinos blancos, 62,5 a 84,6 mg/L en rosado, y 61,0 a $207,0 \mathrm{mg} / \mathrm{L}$ en los vinos tintos. 
El contenido de metanol de algunos tipos de vino de diferentes países europeos se ha informado de la siguiente manera por Cabaroglu (2005): Para los vinos blancos, en Francia, de 38 a $114 \mathrm{mg} / \mathrm{L}$ con un promedio de $60 \mathrm{mg} / \mathrm{L}$, en vinos italianos, de cero a $182 \mathrm{mg} / \mathrm{L}$ con un promedio de $60 \mathrm{mg} / \mathrm{L}$, en vinos de Portugal de cero a $119 \mathrm{mg} / \mathrm{L}$ con un promedio de $63 \mathrm{mg} / \mathrm{L}$. Para el caso de los vinos tintos, en Francia, de 100 a $200 \mathrm{mg} / \mathrm{L}$ con un promedio de $163 \mathrm{mg} / \mathrm{L}$; en vinos italianos, de cero a $635 \mathrm{mg} / \mathrm{L}$ con un promedio de $103 \mathrm{mg} / \mathrm{L}$; en vinos españoles, 39 a $624 \mathrm{mg} / \mathrm{L}$ con un valor medio de $145 \mathrm{mg} / \mathrm{L}$; en vinos portugueses $165 \mathrm{a}$ $264 \mathrm{mg} / \mathrm{L}$ con un promedio de $195 \mathrm{mg} / \mathrm{L}$. Según esto, el contenido de metanol del aperitivo vínico resultado de esta investigación está dentro del rango de contenido metanólico de vinos europeos.

\section{CONCLUSIONES}

Los resultados reportados para los análisis fisicoquímicos, microbiológicos y organolépticos de la pulpa y el producto final estuvieron dentro de los parámetros establecidos por las normas técnicas colombianas (ICONTEC). Se obtuvo una bebida con un contenido alcohólico de $11,3^{\circ} \mathrm{GL}$, lo cual es suficiente para ser considerada como un aperitivo vínico de calidad comparable con la de los productos existentes en el mercado.

\section{REFERENCIAS}

Acosta, A., Perez, F. y Vaillant, F. Chemical characterization, antioxidant properties, and volatile constituents of naranjilla (Solanum quitoense Lam.) cultivated in Costa Rica, Archivos Latinoamericanos de Nutricion, 59, 88-94 (2009).

Arrázola, G., Agroindustrialización del corozo (Bactrisminor), Revista Temas Agrarios: 4(2), 58-64 (1997).

Álvarez, R. y otros tres autores, Caracterización química y sensorial del vino artesanal de tomate de árbol (Cyphomandra betaceae), Revista UDO Agrícola: 9 (2), 436-441 (2009).

A.O.A.C. 9.107/84.972.11/90: Association of oficial analytical chemists (Estados Unidos), Norma americana sobre determinación de Metanol, Maryland (1990).

A.O.A.C. 11.042/84.962.12/90: Association of oficial analytical chemists (Estados Unidos), Norma americana sobre acidez total, Maryland (1990).

A.O.A.C. 11.043/ 84.964.08/90: Association of oficial analytical chemists (Estados Unidos), Norma americana sobre acidez volátil, Maryland (1990).

Bermeo, A., Caracterización de la variabilidad morfológica y algunos atributos químicos de los frutos en una colección de lulo (Solanum quitoense L.) y especies relacionadas de la sección Lasiocarpa, Tesis de titulación, Facultad de ciencias agropecuarias, Universidad Nacional de Colombia, Medellín-Colombia (2005).

Bindon, K. y otros cuatro autores, Relationships between harvest time and wine composition in Vitis vinifera L. cv. Cabernet Sauvignon 1. Grape and wine chemistry. Food Chemistry: 138(2-3), 1696-1705 (2013).

Cabaroglu, T., Methanol contents of Turkish varietal wines and effect of processing, Food Control: 16, 177181 (2005).

Caicedo, O. y B. Higuera, Inducción de polifenoloxidasa en frutos de lulo (Solanum quitoense) como respuesta a la infección con Colletotrichumacutatum, Acta biológica Colombiana: 12, 41-45 (2007).

Camacho, G., Obtención y conservación de pulpas de frutas, Memorias del curso de extensión Universidad Nacional (ICTA), Bogotá- Colombia (1992).

Decreto 3075, Ministro de Salud y Protección Social (Colombia), Aplicación de buenas prácticas de manufactura (BPM), Bogotá-Colombia (1997).

Decretos 365, Ministro de Salud y Protección Social (Colombia), Reglamentación de bebidas alcohólicas en Colombia, Bogotá (1994).

FAO, Fichas técnicas, productos frescos y procesados. Lulo (2006), http://www.fao.org/inpho_archive/content/documents/vlibrary/AE620s/Pfrescos/LULO.HTM. Acceso: Junio 13 (2013). 
Flórez, D.M. y otros cuatro autores, Growth of lulo (Solanum quitoense L.) plants affected by salinity and substrate, Revista Brasileira de Fruticultura: 30, 402-408 (2008).

Fundación Codesarrollo, Alianza Productiva de Lulo (2006), http://www.codesarrollo.org.co/alianzas/alianzas/risaralda/productos/lulo/preinversion/PREINVERSION\%20L ULO\%20DOSQUEBRADAS\%20SANTA\%20ROSA\%20RISARALDA.pdf. Acceso: 11 de Julio de (2013).

Gómez, E. y otros tres autores, Volatile and sensory characterization of red wines from cv. Moravia Agria minority grape variety cultivated in La Mancha region over five consecutive vintages. Food Research International: 44(5), 1549-1560 (2011).

Jiménez, D. y otros siete autores, Interpretation of commercial production information: A case study of lulo (Solanum quitoense), an under-researched Andean fruit, Agricultural System: 104(3), 258-270 (2011).

Lujan, D. y J. Salcedo, Manual de Laboratorio para Biotecnología Alimentaria, 1aㅡ edición, 14-17. Editorial Universidad de Córdoba, Montería, Colombia (2004).

Machado de Castilhos, M., Influence of two different vinification procedures on the physicochemical and sensory properties of Brazilian non-Vitis vinifera red wines, LWT - Food Science and Technology: Available online 1 July 2013 (2013).

Mesas, JM. y M.T. Alegre, El papel de los microorganismos en la elaboración del vino, Cienc. Tecnol. Aliment: 2(4), 174-183 (1999).

NTC 404, Instituto Colombiano de Normas Técnicas y Certificación (Colombia), Establece los requisitos y los métodos de ensayo que deben cumplir los jugos y pulpas de frutas. Quinta Actualización, Bogotá (2007).

NTC 708, Instituto Colombiano de Normas Técnicas y Certificación (Colombia), Norma Colombiana sobre el parámetro de $\mathrm{pH}$ en bebidas alcohólicas en vinos de frutas, 6-10, Bogotá (2005).

NTC 1245, Instituto Colombiano de Normas Técnicas y Certificación (Colombia), Norma Colombiana sobre bebidas alcohólicas. Aperitivos, Quinta Actualización, Bogotá (2004).

Pulido, C.R. y otros tres autores, Node appearance model for Lulo (Solanum quitoense L.) in the high altitude tropics, Biosystems Engineering: 101, 383-387 (2008).

Seung-Joo, L. y otros tres autores, Development of Korean red wines using Vitis labrusca varieties: instrumental and sensory characterization. Food Chemistry: 94(3), 385-393 (2006).

Vogt, E. y otros tres autores, El vino: obtención, elaboración y análisis, 1르 edición, 239 p. Editorial Acribia, Zaragoza, España (1989). 